\title{
Carbapenem Resistance Conferred by OXA-48 in K2-ST86 Hypervirulent Klebsiella pneumoniae, France
}

\author{
Racha Beyrouthy, Guillaume Dalmasso, Aurélien Birer, Frédéric Robin, Richard Bonnet
}

We recovered 2 carbapenem-resistant K2-ST86 hypermucoviscous Klebsiella pneumoniae isolates from patients in France. The isolates had genetic attributes of hypervirulent $K$. pneumoniae but differed in ability to cause mouse lethality. Convergence of hypervirulent $K$. pneumoniae toward resistance could cause a health crisis because such strains could be responsible for severe and untreatable infections.

Klebsiella pneumoniae is a threat to human health Lbecause of the emergence of hypervirulent $K$. pneumoniae, which has caused severe community-acquired infections, and classical multidrug-resistant $K$. pneumoniae involved in hospital outbreaks (1). Classical K. pneumoniae generally lacks the virulence genes associated with invasive diseases (1) and belongs to successful clonal groups, such as sequence type (ST) 11 and ST258 (2). Most hypervirulent K. pneumoniae isolates, which are mainly found in Asia $(3,4)$, belong to the $\mathrm{K} 1$ and $\mathrm{K} 2$ capsular serotypes and are restricted to clonal complexes different from classical multidrug-resistant K. pneumoniae groups, such as K1-ST23, the most prevalent group (2). They rarely harbor acquired antimicrobial resistance genes but have virulence loci and a hypermucoviscous phenotype (5). We describe 2 hypermucoviscous K2-ST86 K. pneumoniae

Author affiliations: Institut National de la Santé et de la Recherche Médicale, Clermont-Ferrand, France (R. Beyrouthy, G. Dalmasso,

F. Robin, R. Bonnet); Centre National de Référence de la Résistance aux Antibiotiques, Clermont-Ferrand (R. Beyrouthy, A. Birer, F. Robin, R. Bonnet); Centre Hospitalier Universitaire, Clermont-Ferrand (R. Beyrouthy, F. Robin, R. Bonnet); Institut National de la Recherche Agronomique (USC-2018),

Clermont-Ferrand (R. Beyrouthy, F. Robin, G. Dalmasso,

R. Bonnet) ; Université Clermont Auvergne, Clermont-Ferrand

(G. Dalmasso, F. Robin, R. Bonnet)

DOI: https://doi.org/10.3201/eid2607.191490 (positive string test) resistant to carbapenems isolated in northern and southern France.

\section{The Study}

In 2017, we recovered the Kpn154 strain from the urine of a 35-year-old man with community-acquired urinary tract infection. He had fever $\left(39^{\circ} \mathrm{C}\right)$ before local symptoms suggesting urinary tract infection caused by bacteremic spread, which was successfully treated with intravenous ceftriaxone. A second strain, Kpn2166, was hospital-acquired and recovered from the feces of a 70-year-old man in the intensive care unit of the hospital at which the 35 -year-old patient was seem. Neither patient reported travel during the past 4 years. Both strains were resistant to all penicillins and their combinations with $\beta$-lactamase inhibitors, and to carbapenems according to EUCAST (European Committee on Antimicrobial Susceptibility Testing) guidelines (https://www.eucast.org) (Table). In addition, Kpn2166 was resistant to the third-generation cephalosporins, quinolones and tigecycline.

We obtained the isolates' whole-genome sequence by hybrid de novo assembly of short and long reads generated with technologies from Illumina (https://www.illumina.com) and Oxford Nanopore (https://nanoporetech.com; European Nucleotide Archive at EMBL-EBI under accession no. PRJEB34867). We typed the isolates as K2-ST86 from whole-genome sequencing using the Institut Pasteur multilocus sequence typing scheme (https://bigsdb.pasteur.fr) and Kleborate (6). Kpn154 harbored carbapenemase-encoding gene $b l a_{\text {oxa-48 }}$ and Kpn2166 the extended-spectrum $\beta$-lactamase-encoding gene bla $a_{\mathrm{CTX}-\mathrm{M}-15}$ as the only acquired $\beta$-lactamase-encoding genes. CTX-M-15 associated with the truncation of the outer membrane protein OmpK36 caused by 11-bp deletion 
Table. Characteristics of carbapenem and hypervirulent Klebsiella pneumoniae isolates from 2 patients, France, 2017*

\begin{tabular}{|c|c|c|}
\hline Characteristic & Kpn154 strain & Kpn2166 strain \\
\hline Patient age, y/sex & $35 / \mathrm{M}$ & $70 / \mathrm{M}$ \\
\hline Sample, context & Urine, community-acquired UTI & Feces, hospital-acquired intestinal carriage \\
\hline \multicolumn{3}{|l|}{$\mathrm{MIC}, \mu \mathrm{g} / \mathrm{mL}$} \\
\hline Ertapenem & 2 & 32 \\
\hline Imipenem & 10 & 10 \\
\hline Meropenem & 2 & 4 \\
\hline Ceftazidime & $0 . \overline{125}$ & $>256$ \\
\hline Ceftriaxone & 0.5 & $>256$ \\
\hline Cefotaxime & 0.5 & $>256$ \\
\hline Cefepime & 0.25 & $>256$ \\
\hline Aztreonam & 0.06 & $>256$ \\
\hline Temocillin & 256 & 32 \\
\hline Tigecyclin & 1 & 4 \\
\hline Colistin & 0.5 & 0.5 \\
\hline Genome size, sequencing depth & $5,555,907 \mathrm{bp}, 120 \times$ & $5,649,836$ bp, $145 x$ \\
\hline Genotype & K2-ST86 & K2-ST86 \\
\hline Resistance replicon, bp & IncL, 100,326 & IncN, 61,761 \\
\hline Resistance marker & bla OXA-48 & bla ${ }_{\mathrm{CTX}-\mathrm{M}-15}, \Delta$ ompK36, $\Delta$ ramR \\
\hline Virulence replicon & IncHI1B/IncFIB, 215,306 & IncHI1B/IncFIB, 226,677 \\
\hline Capsule regulator & $r m p A .2, r m p A 2 \dagger$ & rmpA.2, $\Delta r m p A 2 \ddagger$ \\
\hline Aerobactin-ST§ & AbST1: iucA1B1C1D1iutA1 & AbST1: iucA1B1C1D1iutA1 \\
\hline Salmochelin-ST§ & SmST1: iroB1C1D1N1 & SmST1: iroB1C1D1N1 \\
\hline Yersiniabactin-ST§ & ICEKp3-YbST202LV & ICEKp12like- YbST13LV \\
\hline $\begin{array}{l}\text { “K, capsular genotype; ST, sequence } \\
\text { †New rmpA2 allele. } \\
\text { †Truncated allele harboring a frames }\end{array}$ & $\begin{array}{l}\text { ct infection. } \\
22 \text {. }\end{array}$ & \\
\hline
\end{tabular}

was probably responsible for carbapenem resistance in Kpn2166. In the absence of rpsJ variants previously associated with tigecycline resistance, Kpn2166 resistance to tigecycline and quinolones probably resulted from a frameshift mutation in $\operatorname{ramR}(\mathrm{C} \rightarrow \mathrm{T}$ at position 364) (7).

We identified IncL replicon in Kpn154 and IncN replicon in Kpn2166. The IncL replicon of Kpn154 was a canonical pOXA-48-like plasmid encoding $b l a_{\text {OXA-48 }}$ (GenBank accession no. JN626286). In the Kpn2166 isolate, $b l a_{\text {CTX-M-15 }}$ was encoded by a new ST9-IncN plasmid, included in an IS26-based composite transposon and downstream a truncated ISEcp 1 insertion sequence.

Each isolate harbored an IncHI1B/IncFIB replicon, designated pVIR-Kpn154 and pVIR-Kpn2166 (Table), typified by the reference hvKP virulence plasmid pLVPK (8). They shared with pLVPK 97\% pairwise identity overall and all virulence genes, including the rmpA and rmpA2 genes involved in the hypermucoid phenotype (8). We typed the $\operatorname{rmp} A$ genes as allele 2 according to the Institut Pasteur scheme. However, Kpn2166 rmpA2 harbored in addition a frameshift mutation (coding sequence [CDS] position 196) and 2 other mutations in virulence genes encoding the siderophores (Appendix Figure 1, https://wwwnc.cdc. gov/EID/article/26/7/19-1490-App1.pdf).

We compared the pVIR-Kpn154 and pVIRKpn2166 plasmids with 16 complete hypervirulent K. pneumoniae plasmid sequences (Appendix Table) from the PATRIC database (http:/ / www.patricbrc. org). A single-nucleotide polymorphism-based phylogenic tree showed a link between the major tree branches and the sequence types of hypervirulent $K$. pneumoniae owners but not with their geographic origin (Figure 1, panel A). This finding suggests emergence was more likely caused by spread over distant geographic areas than by local expansion and that limited horizontal transfers between hypervirulent K. pneumoniae isolates probably resulted from the absence of known genes involved in conjugation. Phylogenetic tree analysis also showed 5 groups based on virulence gene synteny, with the predominant group typified by pLVPK (Figure 1, panel B). Because plasmids of ST23-like hypervirulent $K$. pneumoniae all share a similar synteny of virulence genes, ST86 hypervirulent $K$. pneumoniae contains a diversity of plasmid synteny groups (Figure 1, panel A), suggesting that rearrangements of virulence genes occurred several times along plasmid evolution at rearrangement hotspots active in non-ST23 genetic background. For example, pVIR-Kpn2166 and pVIR-Kpn154 differed from reference plasmid pLVPK by the permutations in the $\approx 100-\mathrm{kb}$ region flanked by IS5 mobile elements (Figure 1, panel C). pVIR-Kpn154 contained an additional copy of IS5, which was associated with another $\approx 30-\mathrm{kb}$ permutation + translation event, suggesting that IS5 is a key factor in the evolution and diversity of hypervirulent K. pneumoniae plasmids. 


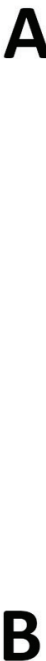

$\begin{array}{lll}\text { QPAG01000004 } & \text { ST23 } & \text { USA } \\ \text { NJPF011000001 } & \text { ST23 } & \text { Hong Kong } \\ \text { CP026166 } & \text { ST3685 } & \text { China } \\ \text { AP006726 } & \text { ST23 } & \text { China } \\ \text { CP019049 } & \text { ST23 } & \text { China } \\ \text { CP025081 } & \text { ST23 } & \text { Singapore } \\ \text { UGM00100000001 } & \text { ST3816 } & \text { UK } \\ \text { LR134257 } & \text { ST23 } & \text { UK } \\ \text { CP028390 } & \text { ST36 } & \text { China } \\ \text { AY378100 } & \text { ST86 } & \text { Taiwan } \\ \text { pVir-Kpn154 } & \text { ST86 } & \text { France } \\ \text { CP026587 } & \text { ST86 } & \text { China } \\ \text { pVir-Kpn2166 } & \text { ST86 } & \text { France } \\ \text { CP030924 } & \text { ST375 } & \text { Korea } \\ \text { CP031258 } & \text { ST66 } & \text { China } \\ \text { CP024708 } & \text { ST65 } & \text { China } \\ \text { CP034776 } & \text { ST86 } & \text { Canada } \\ \text { CP040546 } & \text { ST11 } & \text { China }\end{array}$

pLVPK
219,385 bp
pVIR_Kpn2166
226,677 bp
pVIR_Kpn154
215,306 bp

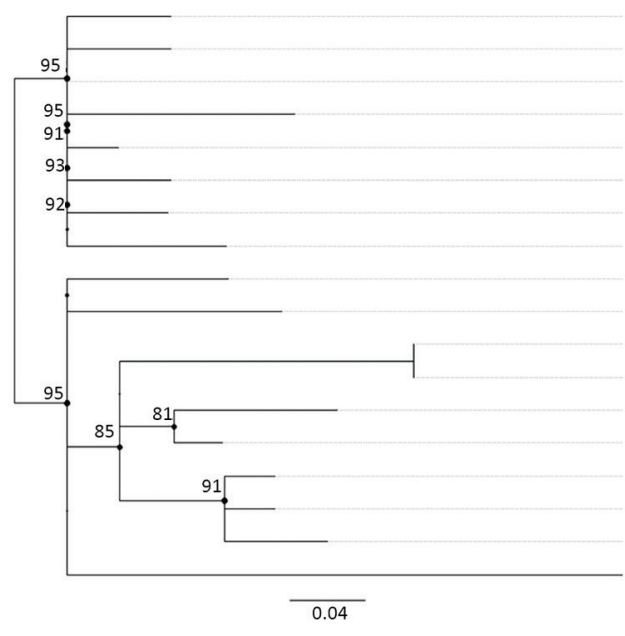

ST3816
ST23
ST23
ST23
ST23
ST23
ST23
ST11
ST65
ST36
ST3685
ST66
ST86
ST86
ST86
ST86
ST86
ST375

UK

China AP006726

UK LR134257

USA QPAG100000

China CP019049

China CP04056

China CP024708

China CP028390

China CP026166

China CP031258

Taiwan AY378100

France

Canada

France

pVir-Kpn2166

CP034776

pVir-Kpn154

China

Korea
Figure 1. Comparison of pVIRKpn2166 and pVIR-Kpn154 Klebsiella pneumoniae isolates from 2 patients in France (bold) with 16 hypervirulent $K$. pneumoniae virulence plasmids recovered from the PATRIC database (http://www. patricbrc.org). A) Single-nucleotide polymorphism-based phylogenetic tree built by RaxML from an alignment generated by BurrowsWheeler Aligner and filtered to remove recombination using Gubbins as previously described (9). The ST and the geographic origin of bacterial hosts are shown. Scale bar indicates mean number of nucleotide substitutions per site. B) Synteny analysis of hypervirulent $K$. pneumoniae virulence plasmids based on data from blastn (https://blast.ncbi.nlm. nih.gov/Blast.cgi). Virulence-based synteny groups are indicated and the operons encoding the virulence factors, virulence synteny groups of plasmids and the ST and the geographic origin of bacterial

2 hosts. Scale bar indicates kbp. C) Comparison of the details of rearrangements observed in $\mathrm{pVIR}$ -

3 Kpn2166 and pVIR-Kpn154 and in pLVPK. Scale bar indicates kbp. ST, sequence type.

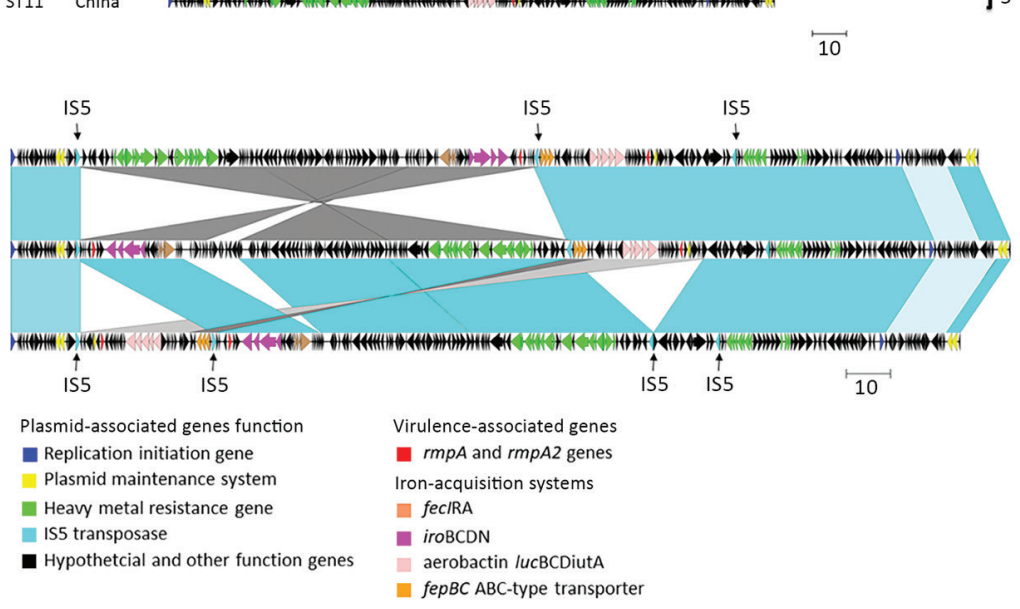

The chromosome of Kpn154 and Kpn2166 exhibited similar organization but differed by 128 insertion/deletion mutations and 1,928 single-nucleotide variants (Appendix Figures 2, 3). The Kpn154 chromosome-mediated ybt virulence locus, which encodes the yersiniabactin, was located in the integrative conjugative element ICEKp3 and was typed ybST202-1LV (6). In Kpn2166, ybt was located in an original isoform of ICEKp12, presenting an $\approx 34-\mathrm{kb}$ deletion compared to the canonical 97,771-bp ICEKp12, and was typed ybST13-1LV (6).

Although Kpn154 and Kpn2166 have the same genetic background and share the same virulence score (6), they also have allelic and synteny differences in virulence genes. We therefore compared the virulence of these isolates in a sepsis model based on outbred 
mice challenged intraperitoneally, as described (Figure 2) (10). Mice injected with $10^{3}$ CFUs of Kpn154 or hypervirulent $K$. pneumoniae reference strain $K$. pneumoniae NTUH-2044 died in $<72 \mathrm{~h}$, in contrast to mice inoculated with Kpn2166 or American Type Culture Collection (ATCC) 13883, showing that only isolate Kpn154 is hypervirulent. Higher bacterial doses $\left(10^{6}\right.$ and $10^{8}$ CFUs) of ATCC13883 and Kpn2166 did not lead to mouse lethality, confirming that Kpn2166 is not hypervirulent in this model, despite harboring all genetic attributes of hypervirulent $K$. pneumoniae except a functional rmpA2 gene and allelic variants of siderophore-encoding genes.

We assessed the production of siderophores in Kpn154, Kpn2166 and the control strains as described (11). Although the siderophore production of the nonvirulent strain ATCC13883 (mean 30.2 + SD 1.8 $\mu \mathrm{g} / \mathrm{mL}$ ) was at the previously reported rate predicting hypervirulent $K$. pneumoniae phenotype $(\geq 30 \mu \mathrm{g} /$ $\mathrm{mL})$, the other strains produced significantly higher siderophore levels $(107.2 \pm 4.5 \mu \mathrm{g} / \mathrm{mL}$ to $306.7 \pm 20.2$ $\mu \mathrm{g} / \mathrm{mL}$; Bonferroni-adjusted $\mathrm{p}=0.0035$ by MannWhitney test), with Kpn154 producing at the lower level (Appendix Figure 4).

\section{Conclusions}

Our results show that a hypermucoviscous K2-ST86 strain can be avirulent in a sepsis mouse model and

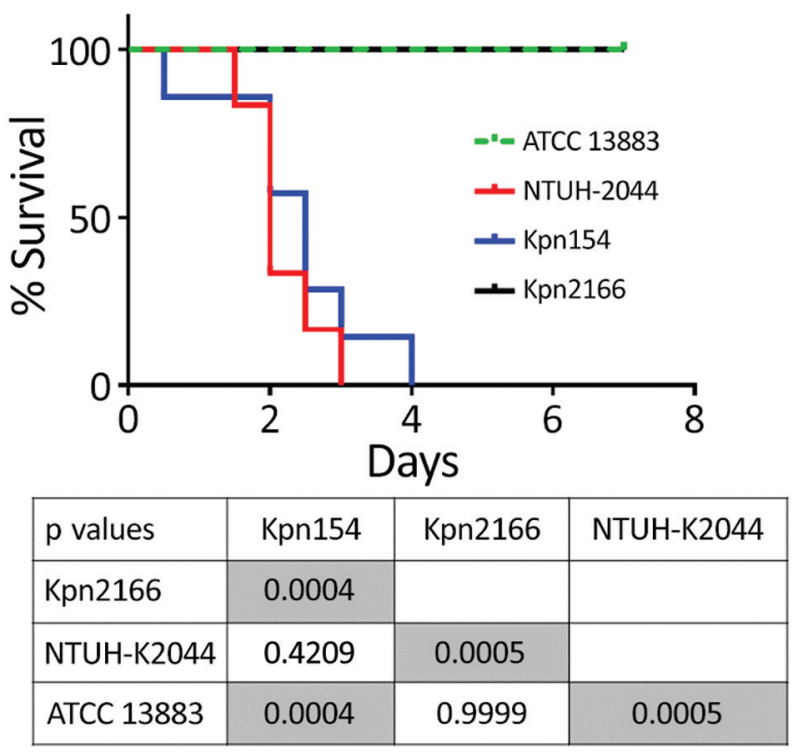

Figure 2. Kaplan-Meier survival curves of mice intraperitoneally challenged with Klebsiella pneumoniae strains Kpn154 and Kpn2166 from 2 patients in France, virulent strain NTUH-K2044, and nonvirulent ATCC 13883 strain, as previously described (10). Mice were injected with $10^{3}$ CFUs and monitored for $96 \mathrm{~h}$. $p$ values were calculated from the Mantel-Cox log rank test for survival curve comparison. Gray shading indicates significant values. ATCC, American Type Culture Collection. that hypervirulence cannot be clearly explained by siderophore production alone. Gene $\operatorname{rmp} A 2$, not required for the hypermucoviscosity phenotype as previously observed (12), might be required for hypervirulent phenotype because it is a main, but not the only, difference we observed between the ST86-K2 strains. Finally, these results highlight the importance of in vivo virulence investigation to identify hypervirulent K. pneumoniae, especially in the absence of an appropriate clinical scenario.

The threat of hypervirulent $K$. pneumoniae acquiring carbapenem resistance is becoming a reality in Asia, especially in China, where hypervirulence prevalence among carbapenem-resistant K. pneumoniae is $7.4 \%-15 \%$ (5). Most resistant isolates are non-K1/K2ST11 and produce carbapenemase KPC-2;. they result from the transfer of the pLVPK-like plasmid into ST11 classical multidrug-resistant K. pneumoniae isolates, as observed in the 2 cases reported outside China (5). Inversely, the carbapenemase-producing isolate Kpn154 results from the transformation of K2-ST86 hypervirulent $K$. pneumoniae by plasmid encoding carbapenemase OXA-48, the most prevalent carbapenemase in France. Similar events occurred with the KPC-2 K2ST86 isolate recently reported in Canada (13) and a few KPC-2 and NDM K1-ST23 cases documented in China and recently in the United States and United Kingdom $(5,14,15)$. The combination of multidrug resistance and enhanced virulence has the potential to trigger the next clinical crisis and cause severe and untreatable infections in previously healthy persons.

\section{Acknowledgments}

We thank Alexis Pontvianne and Lucie Pourpuech for their technical assistance.

This work was supported by the Ministere de l'Education Nationale, de l'Enseignement Superieur et de la Recherche, Institut National de la Santé et de la Recherche Médicale (UMR1071), INRA (USC-2018), Santé Publique France, and the Centre Hospitalier Regional Universitaire de Clermont-Ferrand, France.

\section{About the Author}

Dr. Beyrouthy is a microbiologist in the Associated French National Reference Center for Antibiotic Resistance, Clermont-Ferrand, Clermont-Ferrand University Hospital, and a member of the Microbe, Intestine, Inflammation and Host Susceptibility (M2ISH) research group of the Institut National de la Santé et de la Recherche Médicale. Her primary research interests are antimicrobial drug resistance and Enterobacteriaceae epidemiology. 


\section{References}

1. Meatherall BL, Gregson D, Ross T, Pitout JDD, Laupland KB. Incidence, risk factors, and outcomes of Klebsiella pneumoniae bacteremia. Am J Med. 2009;122:866-73. https:/ / doi.org/10.1016/j.amjmed.2009.03.034

2. Wyres KL, Wick RR, Judd LM, Froumine R, Tokolyi A, Gorrie CL, et al. Distinct evolutionary dynamics of horizontal gene transfer in drug resistant and virulent clones of Klebsiella pneumoniae. PLoS Genet. 2019;15:e1008114. https://doi.org/10.1371/journal.pgen.1008114

3. Prokesch BC, TeKippe M, Kim J, Raj P, TeKippe EME, Greenberg DE. Primary osteomyelitis caused by hypervirulent Klebsiella pneumoniae. Lancet Infect Dis. 2016; 16:e190-5. https://doi.org/10.1016/S1473-3099(16)30021-4

4. Shon AS, Bajwa RPS, Russo TA. Hypervirulent (hypermucoviscous) Klebsiella pneumoniae: a new and dangerous breed. Virulence. 2013;4:107-18. https:/ / doi.org/ 10.4161/viru.22718

5. Lee CR, Lee JH, Park KS, Jeon JH, Kim YB, Cha CJ, et al. Antimicrobial resistance of hypervirulent Klebsiella pneumoniae: epidemiology, hypervirulence-associated determinants, and resistance mechanisms. Front Cell Infect Microbiol. 2017;7:483. https:/ /doi.org/10.3389/ fcimb.2017.00483

6. Lam MMC, Wick RR, Wyres KL, Gorrie CL, Judd LM, Jenney AWJ, et al. Genetic diversity, mobilisation and spread of the yersiniabactin-encoding mobile element ICEKp in Klebsiella pneumoniae populations. Microb Genom. 2018;4(9).

7. Villa L, Feudi C, Fortini D, García-Fernández A, Carattoli A Genomics of KPC-producing Klebsiella pneumoniae sequence type 512 clone highlights the role of RamR and ribosomal S10 protein mutations in conferring tigecycline resistance. Antimicrob Agents Chemother. 2014;58:1707-12. https:/ / doi.org/10.1128/AAC.01803-13

8. Chen YT, Chang HY, Lai YC, Pan CC, Tsai SF, Peng HL Sequencing and analysis of the large virulence plasmid pLVPK of Klebsiella pneumoniae CG43. Gene. 2004;337:189-98. https://doi.org/10.1016/j.gene.2004.05.008
9. Beyrouthy R, Barets M, Marion E, Dananché C, Dauwalder O, Robin F, et al. Novel enterobacter lineage as leading cause of nosocomial outbreak involving carbapenemase-producing strains. Emerg Infect Dis. 2018;24:1505-15. https:/ / doi.org/ 10.3201/eid2408.180151

10. Beyrouthy R, Robin F, Cougnoux A, Dalmasso G, Darfeuille-Michaud A, Mallat H, et al. Chromosomemediated OXA-48 carbapenemase in highly virulent Escherichia coli. J Antimicrob Chemother. 2013;68:1558-61. https://doi.org/10.1093/jac/dkt051

11. Russo TA, Olson R, Fang CT, Stoesser N, Miller M, MacDonald U, et al. Identification of biomarkers for differentiation of hypervirulent Klebsiella pneumoniae from classical K. pneumoniae. J Clin Microbiol. 2018;56:1-12. https://doi.org/10.1128/JCM.00776-18

12. Hsu CR, Lin TL, Chen YC, Chou HC, Wang JT. The role of Klebsiella pneumoniae rmpA in capsular polysaccharide synthesis and virulence revisited. Microbiology. 2011;157: 3446-57. https:/ / doi.org/10.1099/ mic.0.050336-0

13. Mataseje LF, Boyd DA, Mulvey MR, Longtin Y. Two hypervirulent Klebsiella pneumoniae isolates producing a blaKPC-2 carbapenemase from a Canadian patient. Antimicrob Agents Chemother. 2019;63:1-4. https:// doi.org/10.1128/AAC.00517-19

14. Karlsson M, Stanton RA, Ansari U, McAllister G, Chan MY, Sula E, et al. Identification of a carbapenemase-producing hypervirulent Klebsiella pneumoniae isolate in the United States. Antimicrob Agents Chemother. 2019;63:1-6. https://doi.org/10.1128/AAC.00519-19

15. Roulston KJ, Bharucha T, Turton JF, Hopkins KL, Mack DJF. A case of NDM-carbapenemase-producing hypervirulent Klebsiella pneumoniae sequence type 23 from the UK. JMM Case Rep. 2018;5:e005130. https:/ / doi.org/10.1099/ jmmcr.0.005130

Address for correspondence: Racha Beyrouthy, Centre Hospitalier Universitaire, Centre de Biologie, Service de Bactériologie, 58 rue Montalembert, 63000 Clermont-Ferrand, France; email: rbeyrouthy@chu-clermontferrand.fr 